\title{
Pressure Effects on Structural, Electronic, Elastic, and Optical Properties of Cubic and Tetragonal Phases of $\mathrm{BaZrO}_{3}$
}

\author{
Xiong Yang, Ying Wang*, Qinggong Song, Yifei Chen and Yan Hong Xue \\ Institute of Low Dimensional Materials and Technology, College of Science, Civil Aviation University of China, \\ Tianjin 300300, China \\ (Received February 14, 2017; in final form May 24, 2017)

\begin{abstract}
A recent experimental study found the phase transition from cubic to tetragonal phase at $17.2 \mathrm{GPa}$ in $\mathrm{BaZrO}_{3}$. In this paper, we investigate the structural, electronic, elastic, and optical properties of $\mathrm{BaZrO}_{3}$ in the cubic and tetragonal structures under pressure by using first-principle density-functional theory. The main results are summarized as follows: (1) An indirect band structure is displayed for both cubic and tetragonal phases. The band gap increases by $\approx 1 \mathrm{eV}$ as a result of the phase transition. (2) The elastic constants $C_{i j}$ 's indicate that the cubic and tetragonal phases are mechanically stable. The easiest deformation mechanism in both phases is the shear along the (100) plane. (3) The real and imaginary parts of dielectric function for the cubic and tetragonal phases shift towards higher energies with pressure. Our results are in agreement with the available experimental and prior theoretical data.
\end{abstract}

DOI: 10.12693/APhysPolA.133.1138

PACS/topics: pressure effect, electronic structure, elastic property, optical property

\section{Introduction}

Barium zirconate $\left(\mathrm{BaZrO}_{3}\right)$ with a cubic perovskite structure, a classic $\mathrm{ABO}_{3}$ compound, has been a popular target because it displays a large number of desired physical properties, such as a high-temperature proton conductor [1], a useful dielectric material for wireless communications [2], a good substrate in thin-film deposition [3] and solid oxide fuel cells [4]. The $\mathrm{ABO}_{3}$ crystals of cubic perovskite structure exhibit rich structural phase transitions due to the changes of external conditions, such as pressure or temperature. For instance, Köhler et al. [5] predicted theoretically a phase transition from cubic to tetragonal structure in $\mathrm{EuTiO}_{3}$, and subsequently verified in experiment that it occurs at $T=282 \mathrm{~K}$. Recently, high pressure experimental discoveries have shown that $\mathrm{SrTiO}_{3}$ and $\mathrm{BaZrO}_{3}$ undergo a structural phase transition from cubic to tetragonal phase, respectively $[6,7]$. The phase transformation is generally considered to have the same driving force from symmetry breaking, i.e., the phonon mode instability manifested clearly by imaginary frequency optical modes at the $R$-point in the phonon spectrum [8-13].

It is well known that the electronic, elastic, and optical properties of $\mathrm{ABO}_{3}$ compounds under external pressure will be altered with the phase transition, because of the change of the relative positions of atoms and interatomic distances. Such effects of pressure on the properties of compounds are not only attractive in physics, but also intriguing in technology. However, to the best of our knowledge, there is no systematic research on the electronic, elastic, and optical properties of $\mathrm{BaZrO}_{3}$ in the

*corresponding author; e-mail: $\mathrm{x}$-yang@cauc.edu.cn cubic and tetragonal phases under pressure. In this paper, we explore how high pressure affects the structural, electronic, elastic, and optical properties of $\mathrm{BaZrO}_{3}$ in the cubic and tetragonal phases by performing extensive first-principle calculations. Our work provides a comprehensive perspective into the pressure effects on the fundamental properties of $\mathrm{BaZrO}_{3}$.

\section{Computational details}

The first-principle calculation has been successful in accurate understanding of the structural stability, the physical properties of various compounds including perovskite oxide, yttrium orthoaluminate, rhodium silicides, and carbon dioxide, under external conditions (pressure or impurity) [14-17]. Our first-principles calculations are performed using density functional theory (DFT) within the plane-wave basis available in CASTEP code [18]. We used the generalized gradient approximation (GGA) in the scheme of Perdew-Wang 91 (PW91) [19]. The valence electron configurations for $\mathrm{Ba}, \mathrm{Zr}, \mathrm{O}$ atoms are dealt with $2 s^{2} 2 p^{4}, 5 s^{2} 5 p^{6} 6 s^{2}$ and $4 s^{2} 4 p^{6} 4 d^{2} 5 s^{2}$, respectively. The tolerances of stress, force and self-consistent field are $0.02 \mathrm{GPa}, 0.01 \mathrm{eV} / \AA$ and $5.0 \times 10^{-7} \mathrm{eV} /$ atom, respectively. Ultrasoft pseudopotentials within a $775 \mathrm{eV}$ cut-off energy are chosen to optimize structure. The $k$ mesh is sampled according to a Monkhorst-Pack scheme with a spacing of $0.04 \AA$. Such parameters have been tested to be sufficient for convergence $(<1 \mathrm{meV})$.

The phase transition from cubic to tetragonal phase was observed at $17.2 \mathrm{GPa}$ in $\mathrm{BaZrO}_{3}$ [7]. We use the experimental lattice constants $a=4.194 \AA$ at $0 \mathrm{GPa}$ in the cubic phase, and $a=5.5074 \AA, c=7.9273 \AA$ at $46.4 \mathrm{GPa}$ in the tetragonal phase [7]. As summarized in Tables I and II, our optimized lattice parameters as well as internal atomic positions agree well with the experimental and other theoretical results. 
TABLE I

The relaxed structure lattice constants $[\AA]$, elastic constants, bulk modulus $B$, shear modulus $G$ and the Young modulus $E[\mathrm{GPa}]$ of $\mathrm{BaZrO}_{3}$ in cubic phase at $0 \mathrm{GPa}$ compared with the experimental data and prior theoretical results.

\begin{tabular}{c|c|c|c|c|c|c|c}
\hline \hline Source & $a$ & $C_{11}$ & $C_{12}$ & $C_{44}$ & $B$ & $G$ & $E$ \\
\hline ours & 4.223 & 295 & 82 & 85 & 153 & 93 & 260 \\
expt. & 4.192 & - & - & - & $189 \pm 26^{a}$ & $103^{B}$ & $243^{B}$ \\
GGA $^{c}$ & 4.207 & 322.5 & 79 & 70 & 157 & 91 & 229 \\
LDA $^{d}$ & 4.154 & 336 & 95 & 89.5 & 175.3 & 102 & 256
\end{tabular}

TABLE II

Lattice constants $[\AA]$ and atom positions in tetragonal phase of $\mathrm{BaZrO}_{3}$ at $46.4 \mathrm{GPa}$, computational and experimental (Ref. [7]) data.

\begin{tabular}{c|c|c|c|c|c|c}
\hline \hline Source & $a$ & $c$ & $\mathrm{Ba}$ & $\mathrm{Zr}$ & $\mathrm{O})$ & $\mathrm{O}(2)$ \\
\hline ours & 5.521 & 8.027 & $(0,0.5,0.25)$ & $(0,0,0)$ & $(0,0,0.25)$ & $(0.2988,0.7988,0)$ \\
expt. & 5.507 & 7.927 & $(0,0.5,0.25)$ & $(0,0,0)$ & $(0,0,0.25)$ & $(0.2181,0.7131,0)$
\end{tabular}

\section{Results and discussion}

\subsection{Structural properties}

The experimental studies confirmed that a phase transition from cubic $(P m \overline{3} \mathrm{~m})$ to tetragonal $(\mathrm{I} 4 / \mathrm{mcm})$ phase takes place in $\mathrm{BaZrO}_{3}$ under compression. The similar transition behavior was observed in the other cubic perovskites, such as $\mathrm{SrTiO}_{3}$ and $\mathrm{EuTiO}_{3}[5,6]$. It is suggested that the cubic-tetragonal phase transition resulted from the phonon mode instability with the rotation of oxygen octahedra around [001] under high pressure [813]. As shown in Fig. 1, our calculation presents the pressure dependence of $\mathrm{ZrO}_{6}$ octahedra rotation angle in $\mathrm{BaZrO}_{3}$. The oxygen octahedra rotates by $6.396^{\circ}$ (corresponding to a $\mathrm{Zr}-\mathrm{O}-\mathrm{Zr}$ angle of $167.2^{\circ}$ ) as a result of the cubic-tetragonal phase transition at 17.2 GPa. The octahedral rotation angle $\theta$ increases with increasing pressure, and the extremely similar dependence of $c / a$ on pressure can be found. The change rate of rotation angle and $c / a$ decreases abruptly at $28 \mathrm{GPa}$, which implies that a displacive phase transition comes up at this pressure according to the elastic properties shown in Fig. 5. Our optimized tetragonal structure with $\mathrm{ZrO}_{6}$ octahedra tilting is only due to the rotations of $\mathrm{ZrO}_{6}$ octahedra along the $c$ direction, without any tilting along the $a$ and $b$ directions, which is in agreement with the experimental observation [7].

\subsection{Electronic properties}

The energy band structure and projected density of states near the Fermi energy for the cubic and tetragonal phases of $\mathrm{BaZrO}_{3}$ at $6 \mathrm{GPa}$ and $35 \mathrm{GPa}$ are shown in Figs. 2 and 3 . The overall band profiles at other pressure for the two phases are found to be similar to Figs. 2 and 3. The Brillouin zone shape of the cubic phase differs from that of the tetragonal phase, hence the selected high symmetry points along the transverse axis in Fig. 2
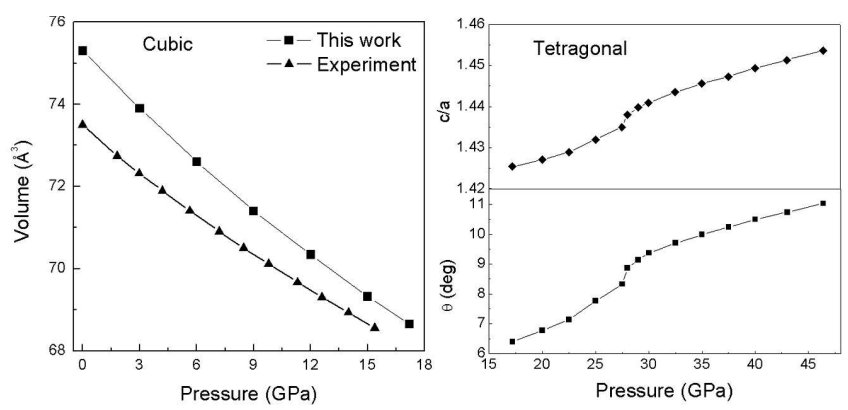

Fig. 1. Pressure dependence of the unit cell volume in the cubic phase, $c / a$ and octahedral rotation degree $\theta$ in the tetragonal phase of $\mathrm{BaZrO}_{3}$. The acceptable difference between the theoretical and experimental volumes in the cubic phase is due to the slightly exaggerated lattice constant as a result of GGA method.

distinguish from those in Fig. 3. For both the phases, the top of the valence band mostly consists of O $2 p$ and $\mathrm{Zr} 4 d$ electronic states, while $\mathrm{Zr} 4 d$ states mainly dominate the bottom of the conduction band. The energy gap for the cubic phase is indirect along the $R \rightarrow \Gamma$ direction. For the tetragonal phase, the minimum of the conduction band is at $\Gamma$, and the maximum of the valence band is at the high symmetry point $(0,0.20,0)$ along the $X(0,0.5,0)$ to $\Gamma$ direction. The energy at the point $(0,0.20,0)$ is only $0.045 \mathrm{eV}$ higher than that at $\Gamma$, indicated by the arrow in Fig. 3.
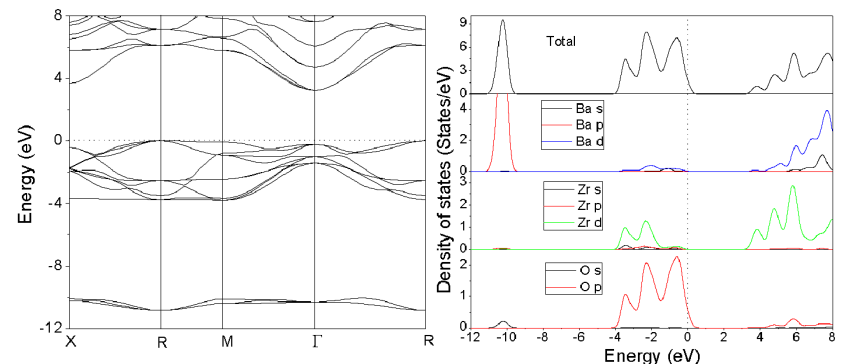

Fig. 2. Electronic band structure (left part) and projected density of states (right part) for the cubic phase of $\mathrm{BaZrO}_{3}$ under $6 \mathrm{GPa}$.
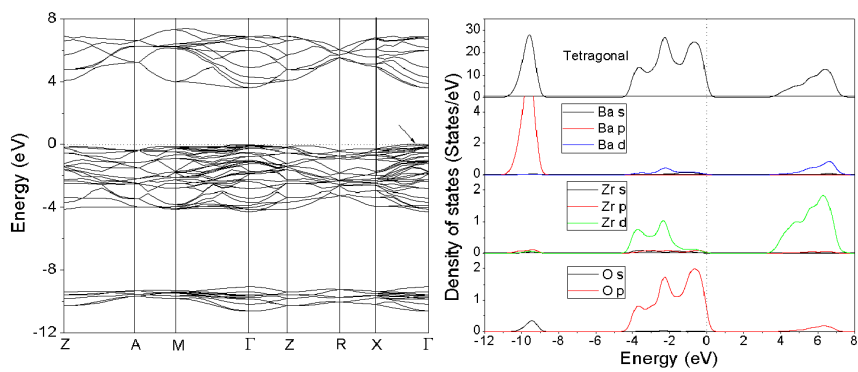

Fig. 3. Electronic band structure (left part) and projected density of states (right part) for the tetragonal phase of $\mathrm{BaZrO}_{3}$ under $35 \mathrm{GPa}$.

Figure 4 indicates the pressure-induced band gap variation for $\mathrm{BaZrO}_{3}$. Our calculated band gap at equilib- 


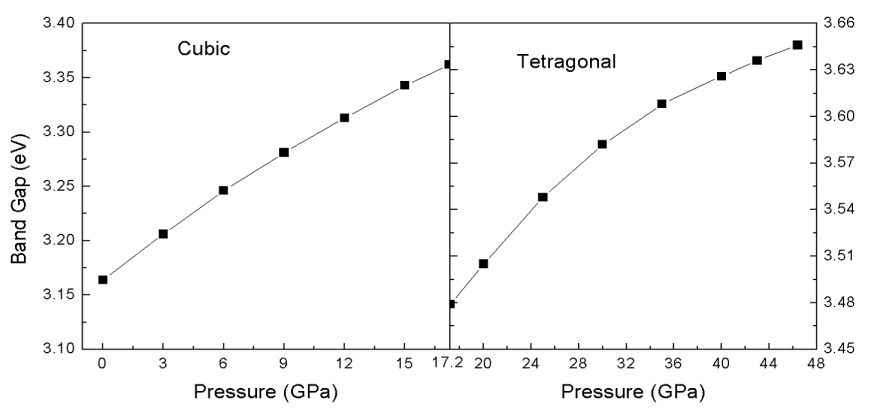

Fig. 4. Band gap of the cubic and tetragonal phases of $\mathrm{BaZrO}_{3}$ as a function of pressure.

rium is $3.164 \mathrm{eV}$, which can be compared to the theoretical value of $3.23 \mathrm{eV}$ [21]. The theoretical values using GGA method are underestimated with respect to the experimental value of $5.33 \mathrm{eV}$ [23]. Moreover, GGA usually agrees with the more time-consuming HeydScuseria-Ernzerhof (HSE06) on pressure coefficients of band gap [24-26]. Also, the different exchange correlation functionals appear not to change the trends in the dependence of the band gap on pressure. Thus, pressure coefficients of the band gap computed using DFT could be observed in experiment. As shown in Fig. 4, the band gap energy increases by $\approx 1 \mathrm{eV}$ while phase transition from cubic to tetragonal occurring at 17.2 GPa, because of the decrease of conduction band width. In both the phases, the pressure results in the linear increase of the band gap. This pressure effect is very similar to that of $\mathrm{SrTiO}_{3}$ in Ref. [27]. The change rate of the band gap for the tetragonal phase drops gradually with increase of pressure. We note that while compression increases from 17.2 $\mathrm{GPa}(0 \mathrm{GPa})$ to $46.4 \mathrm{GPa}(17.2 \mathrm{GPa})$ in the tetragonal (cubic) phase, the band gap increases only by $4.8 \%(6.3 \%)$.

\subsection{Elastic properties}

The elastic constants describe the nature character of the forces operating in solids and provide information on the stiffness and stability of materials. Based on the fully structure relaxation under pressure in the cubic and tetragonal phases, the elastic tensor $C_{i j}$ is calculated by using the "volume-conserving technique". We define the bulk modulus and shear modulus by the Reuss theory $\left(B_{R}, G_{R}\right)$ and the Voigt theory $\left(B_{V}, G_{V}\right)$ respectively [28]. In the commonly used Voigt approximation,

$$
\begin{aligned}
& B_{V}=\frac{1}{9}\left[\left(C_{11}+C_{22}+C_{33}\right)+2\left(C_{12}+C_{23}+C_{31}\right)\right], \\
& G_{V}=\frac{1}{15}\left[\left(C_{11}+C_{22}+C_{33}\right)-\left(C_{12}+C_{23}+C_{31}\right)\right. \\
& \left.\quad+3\left(C_{44}+C_{55}+C_{66}\right)\right] .
\end{aligned}
$$

On the other hand, according to the Reuss approximation,

$$
\frac{1}{B_{R}}=\left(S_{11}+S_{22}+S_{33}\right)+2\left(S_{12}+S_{23}+S_{31}\right),
$$

$$
\begin{aligned}
& \frac{15}{G_{R}}=4\left(S_{11}+S_{22}+S_{33}\right)-4\left(S_{12}+S_{23}+S_{31}\right) \\
& \quad+3\left(S_{44}+S_{55}+S_{66}\right)
\end{aligned}
$$

where $C_{i j}$ and $S_{i j}$ describe the elastic stiffness and the elastic compliance, respectively. In addition, the socalled Hill theory [28] gives

$$
\begin{aligned}
& B=\left(B_{V}+B_{R}\right) / 2, \\
& G=\left(G_{V}+G_{R}\right) / 2 .
\end{aligned}
$$

the Young modulus $(E)$ and the Poisson ratio $(\gamma)$ are obtained by the relation

$$
\begin{aligned}
& E=9 B G /(3 B+G), \\
& \gamma=(3 B-2 G) /[2(3 B+G)] .
\end{aligned}
$$

The elasticity of a cubic crystal is characterized by three independent modulus parameters: $C_{11}, C_{12}$ and $C_{44}$. The symmetry of the tetragonal phase is below that of the cubic phase, hence the independent elastic constants increase to $C_{11}, C_{12}, C_{13}, C_{33}, C_{44}$ and $C_{66}$. The calculated elastic constants of $\mathrm{BaZrO}_{3}$ at equilibrium are represented in Table I. Our results at zero pressure are in good agreement with previous calculations and experimental data. Figure 5 shows the pressure effects on the elastic constants in the cubic and tetragonal phases. The cubic phase within 0-17.2 GPa and the tetragonal phase within 17.2-46.4 GPa are mechanically stable, because the $C_{i j}$ matrix satisfies the well-known Born stability criteria [29]. For cubic phase, they are

$$
C_{11}>0, C_{11}>C_{12}, C_{44}>0 \text {. }
$$

For tetragonal phase, they are

$$
\begin{aligned}
& C_{11}>0, C_{33}>0, C_{44}>0, C_{66}>0, C_{11}>C_{12}, \\
& C_{11}+C_{33}-2 C_{13}>0, \\
& 2\left(C_{11}+C_{12}\right)+C_{33}+4 C_{13}>0 .
\end{aligned}
$$

The cubic elastic coefficients $C_{11}, C_{12}$ and the tetragonal coefficients $C_{11}, C_{12}, C_{13}, C_{33}$ increase with pressure, while the cubic $C_{44}$ and the tetragonal $C_{44}, C_{66}$ do not vary much with pressure. The curves of cubic $C_{11}, C_{12}$ and tetragonal $C_{11}, C_{12}, C_{13}, C_{33}$ show a leap at about $12 \mathrm{GPa}$ and $28 \mathrm{GPa}$ respectively, which indicates that a displacive phase transition occurs at these pressures. The similar feature has been observed in the pressure effects of the elastic constants of $\mathrm{SrTiO}_{3}$ in Ref. [30]. The $C_{44}$ constant is almost unchanged in both cubic and tetragonal phases, implying that the shear along the (100) plane is the easiest and does not depend on the phase. The tetragonal $C_{11}$ is much smaller than $C_{33}$, which means that $a$ and $b$ axes in this phase are easier to be compressed than in the cubic one.

Based on the obtained elastic coefficients of $\mathrm{BaZrO}_{3}$, we further predict its noteworthy elastic properties in the cubic and tetragonal phases, such as bulk modulus, shear modulus, the Young modulus, the Poisson ratio, and anisotropy factor. The calculated results are summarized in Tables III and IV. It can be seen that the three moduli increase as pressure increases, and the Young modulus $E$ is always largest, shear modulus $G$ smallest. It is well 
TABLE III

Bulk modulus $B$, shear molulus $G$, the Young modulus $E$, ([GPa]), the Poisson ratio $\gamma$, and anisotropy factor $A^{U}$ of $\mathrm{BaZrO}_{3}$ in cubic phase at several pressures.

\begin{tabular}{c|c|c|c|c|c|c}
\hline \hline$P$ & $B$ & $G$ & $E$ & $B / G$ & $\gamma$ & $A^{U}$ \\
\hline 0 & 153 & 93 & 260 & 1.645 & 0.217 & 0.063 \\
3 & 165 & 98 & 282 & 1.684 & 0.214 & 0.102 \\
6 & 187 & 102 & 308 & 1.833 & 0.225 & 0.145 \\
9 & 203 & 107 & 331 & 1.897 & 0.228 & 0.187 \\
12 & 197 & 111 & 346 & 1.775 & 0.207 & 0.231 \\
15 & 208 & 115 & 368 & 1.809 & 0.206 & 0.278 \\
17.2 & 227 & 118 & 386 & 1.924 & 0.216 & 0.308
\end{tabular}

TABLE IV

Bulk modulus $B$, shear molulus $G$, the Young modulus $E$, ([GPa]), the Poisson ratio $\gamma$, and anisotropy factor $A^{U}$ of $\mathrm{BaZrO}_{3}$ in tetragonal phase at several pressures.

\begin{tabular}{c|c|c|c|c|c|c}
\hline \hline$P$ & $B$ & $G$ & $E$ & $B / G$ & $\gamma$ & $A^{U}$ \\
\hline 17.2 & 223 & 115 & 294 & 1.939 & 0.280 & 0.212 \\
20 & 234 & 118 & 303 & 1.983 & 0.284 & 0.234 \\
22.5 & 243 & 120 & 309 & 2.025 & 0.288 & 0.263 \\
25 & 251 & 120 & 310 & 2.092 & 0.294 & 0.2344 \\
30 & 265 & 118 & 308 & 2.246 & 0.306 & 0.2556 \\
35 & 287 & 124 & 325 & 2.315 & 0.311 & 0.297 \\
40 & 305 & 126 & 332 & 2.420 & 0.318 & 0.321 \\
46.4 & 326 & 128 & 340 & 2.547 & 0.326 & 0.357
\end{tabular}

known that the Young modulus is a crucial parameter for selecting materials in engineering design. The material is stiffer for the larger the Young modulus. Our calculations indicate that stiffness of $\mathrm{BaZrO}_{3}$ becomes higher and higher with increase of pressure for both the cubic and tetragonal phases. the Poisson ratio $\gamma$ can describe the characteristic of the bonding forces to some extent. $\gamma=0.5$ is the lower limit for central-force solids and 0.5 is the upper limit, which corresponds to infinite elastic anisotropy [31]. The obtained values of the cubic phase within the whole range of $0-17.2 \mathrm{GPa}$ are below 0.25 , however, the values of the tetragonal phase are above 0.25 up to $46.4 \mathrm{GPa}$. This indicates that the interatomic forces of $\mathrm{BaZrO}_{3}$ are non-central for the cubic phase, but central for the tetragonal phase. In order to evaluate the ductile/brittle nature of materials, Pugh [32] proposed a criterion. When the ratio of bulk to shear modulus $B / G$ is bigger (less) than 1.75, the material is considered ductile (brittle). From the predicted values of $B / G$, it can be judged that $\mathrm{BaZrO}_{3}$ in the cubic phase is brittle below $6 \mathrm{GPa}$, ductile above $6 \mathrm{GPa}$. The ductility of $\mathrm{BaZrO}_{3}$ in the tetragonal phase is more and more significant with increase of pressure.

The evaluation of elastic anisotropy in material is of great significance to understand the mechanical properties of crystal. An appropriate measure to quantify the degree of anisotropy is necessary. Ranganathan and Ostoja-Starzewski [33] developed a universal anisotropy index $A^{U}$ to estimate the anisotropic characteristic, which is as follows:

$$
A^{U}=\frac{5 G_{V}}{G_{R}}+\frac{B_{V}}{B_{R}}-6 .
$$

For an isotropic crystal, $A^{U}=0$. The departure of $A^{U}$ from zero defines the degree of elastic anisotropy. The calculated results of $A^{U}$ in Tables III and IV deviate slightly from 0 , indicating the relatively small elastic anisotropy characteristic of $\mathrm{BaZrO}_{3}$ in the cubic and tetragonal phases. It is notable that the increasing pressure leads to more and more heavy extent of anisotropy for both cubic and tetragonal phases, except for the leap at about $25 \mathrm{GPa}$ in the tetragonal phase.

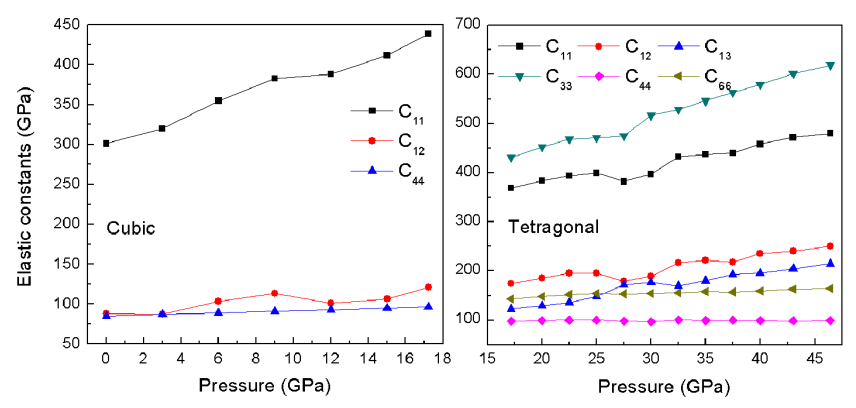

Fig. 5. Elastic constants of the cubic and tetragonal phases of $\mathrm{BaZrO}_{3}$ as a function of pressure.

\subsection{Optical properties}

The dielectric function $\varepsilon(\omega)$ can be used to describe the linear response of the system to electromagnetic radiation, which is connected with the interaction between photons and electrons [34]. The imaginary part $\varepsilon_{2}(\omega)$ can be calculated from the momentum matrix elements between the occupied and unoccupied electronic states within selection rules, and the real part $\varepsilon_{1}(\omega)$ is linked with $\varepsilon_{2}(\omega)$ by the Kramer-Krönig relations. The other linear optical parameters, such as refractive index and electron energy-loss coefficient, can be evaluated on the basis of $\varepsilon_{1}(\omega)$ and $\varepsilon_{2}(\omega)$. To check the calculation reliability with using GGA-PW91 and ultrasoft pseudopotential scheme, we also calculated $\varepsilon_{1}(\omega)$ and $\varepsilon_{2}(\omega)$ of the cubic $\mathrm{SrTiO}_{3}$ with perovskite structure similar to $\mathrm{BaZrO}_{3}$, which agree well with the experimental data, as shown in Fig. 6. In our calculations of optical spectra, a sufficiently dense $k$-mesh is applied by the separation of optical $k$ points $0.021 / \AA$.

The real and imaginary parts of dielectric function of $\mathrm{BaZrO}_{3}$ in the cubic and tetragonal phases under pressure are displayed in Figs. 7 and 8. Since the tetragonal phase of $\mathrm{BaZrO}_{3}$ is optically anisotropic, components of $\varepsilon_{1}(\omega)$ and $\varepsilon_{2}(\omega)$ corresponding to the polarization vectors perpendicular $(E \perp c)$ and parallel $(E \| c)$ to the crystallographic $c$ axis have been considered. Figures 7 and 8 only shows the curves with $E \perp c$, because the curve structures of $E \perp c$ and $E \| c$ are similar to each other. The whole $\varepsilon_{1}(\omega)$ and $\varepsilon_{2}(\omega)$ curves for two phases shift towards higher energies with increase of pressure, showing blueshift compared to the equilibrium state. The first 


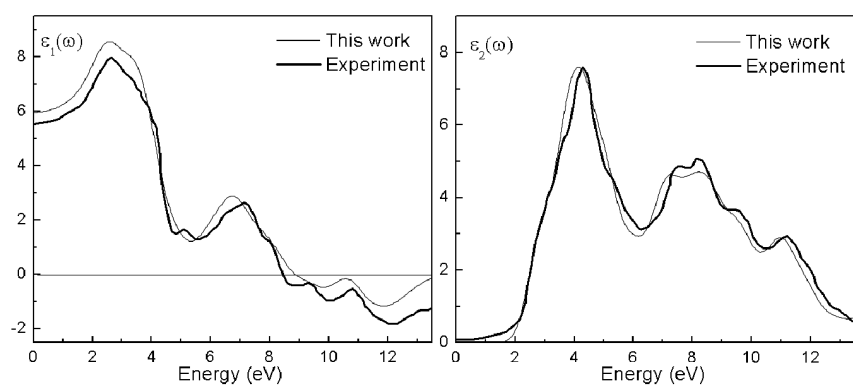

Fig. 6. Real and imaginary parts of dielectric function $\varepsilon(\omega)$ of $\mathrm{SrTiO}_{3}$. The experimental data are from Ref. [35].

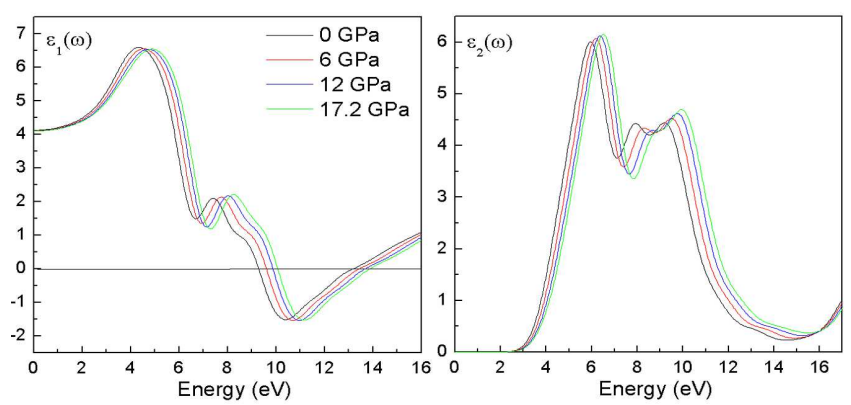

Fig. 7. Real and imaginary parts of dielectric function $\varepsilon(\omega)$ of the cubic phase of $\mathrm{BaZrO}_{3}$ as a function of pressure.

peak of $\varepsilon_{2}(\omega)$ originates from the transfer of the highest valence electrons ( $\mathrm{O} p$ states) to the lowest conduction bands (Zr $d$ states). The shift of the first peak implies the enlargement of the band gap resulted by pressure, which is consistent with Fig. 4. In terms of the low-frequency behavior of the dielectric function, the static dielectric constant $\varepsilon_{1}(0)$ and zero frequency refractive index $n(0)$ are of interest in experiment.

As displayed in Figs. 9 and 10, the transition from cubic to tetragonal phase reduces the values of static dielectric constant $\varepsilon_{1}(0)$ and refractive index $n(0)$. The pressure effects lead to the decrease (increase) of $\varepsilon_{1}(0)$ and $n(0)$ for the cubic (tetragonal) phase, however, the drop (raise) is rather small compared to the initial value. It is worthwhile to note that the $n(0)$ of the ordinary po-

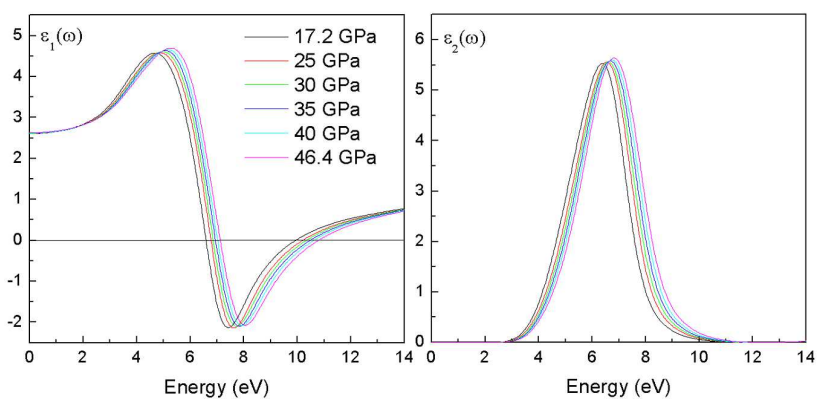

Fig. 8. Real and imaginary parts of dielectric function $\varepsilon(\omega)$ of the tetragonal phase of $\mathrm{BaZrO}_{3}$ as a function of pressure.

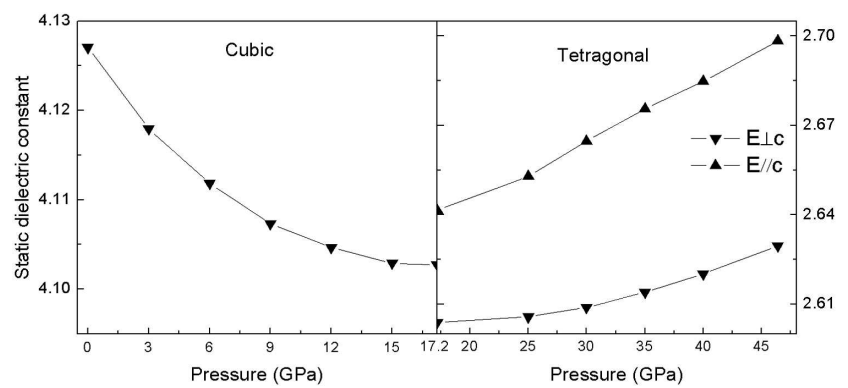

Fig. 9. Static dielectric constant of the cubic and tetragonal phases of $\mathrm{BaZrO}_{3}$ as a function of pressure.

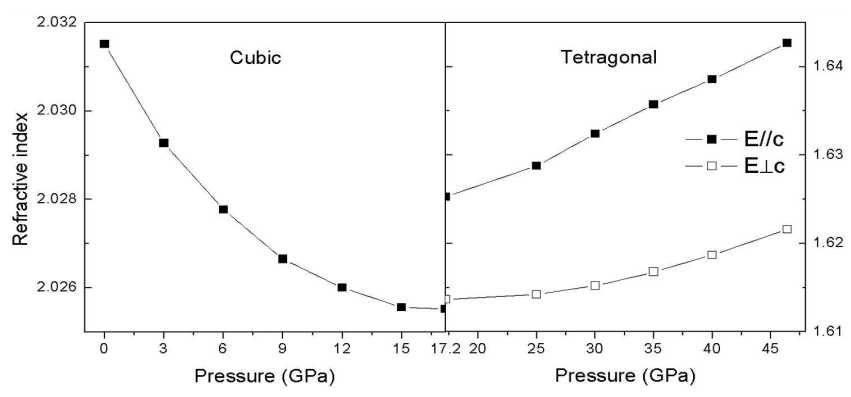

Fig. 10. Refractive index of the cubic and tetragonal phases of $\mathrm{BaZrO}_{3}$ as a function of pressure.

larization is smaller than that of the extraordinary polarization for the tetragonal phase (right part of Fig. 10), and $\mathrm{BaZrO}_{3}$ compound is positive uniaxial crystal.

\section{Conclusions}

In this paper, we present comprehensive first-principle calculation results about the pressure effects on the fundamental properties of $\mathrm{BaZrO}_{3}$ in the cubic and tetragonal structures for the first time. The band gap for the cubic and tetragonal phases is indirect, and it increases by $\approx 1 \mathrm{eV}$ while the phase transition occurs. The elastic constants $C_{i j}$ 's indicate that the cubic and tetragonal phases are stable. The easiest deformation mechanism in both phases is the shear along the (100) plane. The real and imaginary parts of dielectric function for the cubic and tetragonal phases shift towards higher energies with pressure. The pressure effects on the other elastic and optical parameters, including bulk modulus, shear modulus, the Young modulus and refractive index are also presented.

\section{Acknowledgments}

This work was supported by the Fundamental Research Funds for the Central Universities (3122014K003).

\section{References}

[1] K.D. Kreuer, Annu. Rev. Mater. Res. 33, 333 (2003).

[2] M.T. Sebastian, Dielectric Materials for Wireless Communication, Elsevier, Amsterdam 2008. 
[3] P.S. Dobal, A. Dixit, R.S. Katiyar, Z. Yu, R. Guo, A.S. Bhalla, J. Appl. Phys. 89, 8085 (2001).

[4] M. Miura, B. Maiorov, T. Kato, T. Shimode, K. Wada, S. Adachi, K. Tanabe, Nat. Commun. 4, 2499 (2013).

[5] J. Köhler, R. Dinnebier, A. Bussmann-Holder, Phase Transit. 85, 949 (2012).

[6] M. Guennou, P. Bouvier, B. Krikler, J. Kreisel, D. Machon, Phys. Rev. B 81, 054115 (2010).

[7] X. Yang, Q.J. Li, R. Liu, B. Liu, H.F. Zhang, S.Q. Jiang, J. Liu, B. Zou, T. Cui, B.B. Liu, J. Appl. Phys. 115, 124907 (2014).

[8] K.Z. Rushchanskii, N.A. Spaldin, M. Lezaic, Phys. Rev. B 85, 104109 (2012).

[9] J.W. Bennett, I. Grinberg, A.M. Rappe, Phys. Rev. B 73, 180102 (2006).

[10] A. Bilic, J.D. Gale, Phys. Rev. B 79, 174107 (2009).

[11] A.I. Lebedev, I.A. Sluchinskaya, Phys. Solid State 55 , 1941 (2013)

[12] A.R. Akbarzadeh, I. Kornev, C. Malibert, L. Bellaiche, J.M. Kiat, Phys. Rev. B 72, 205104 (2005).

[13] W. Zhong, D. Vanderbilt, Phys. Rev. Lett. 74, 2587 (1995).

[14] Cheng Lu, Jingjing Wang, Ping Wang, Xinxin Xia, Yuanyuan Jin, Peifang Lie, Gang Bao, Phys. Chem. Chem. Phys. 19, 1420 (2017).

[15] Meng Ju, Cheng Lu, Yauyuen Yeung, Xiaoyu Kuang, Jingjing Wang, Yongsheng Zhu, ACS Appl. Mater. Inter. 8, 30422 (2016).

[16] Jing-Jing Wang, A. Hermann, Xiao-Yu Kuang, YuanYuan Jin, Cheng Lu, Chuan-Zhao Zhang, Meng Ju, Meng-Ting Sic, Toshiaki Iitaka, RSC Adv. 5, 53497 (2015).

[17] Cheng Lu, Maosheng Miao, Yanming Ma, J. Am. Chem. Soc. 135, 14167 (2013).
[18] V. Milman, B. Winkler, J.A. White, C.J. Packard, M.C. Payne, E.V. Akhmatskaya, R.H. Nobes, Int. J. Quantum Chem. 77, 895 (2000).

[19] J.P. Perdew, K. Burke, M. Ernzerhof, Phys. Rev. Lett. 77, 3865 (1996).

[20] S. Yamanaka, H. Fujikane, T. Hamaguchi, H. Muta, T. Oyama, T. Matsuda, S. Kobayashi, K. Kurosaki, J. Alloys Comp. 359, 109 (2003).

[21] R. Terki, H. Feraoun, G. Bertrand, H. Aourag, Phys. Status Solidi B 242, 1054 (2005).

[22] R.D. King-Smith, D. Vanderbilt, Phys. Rev. B 49, 5828 (1994)

[23] J. Robertson, J. Vac. Sci. Technol. B 18, 1785 (2000).

[24] L. Qin, Y. Duan, H. Shi, L. Shi, G. Tang, J. Phys. Condens. Matter 25, 045801 (2013).

[25] Yifeng Duan, Lixia Qin, Liwei Shi, Gang Tang, Hongliang Shi, Appl. Phys. Lett. 100, 022104 (2012).

[26] Dong Lv, Yifeng Duan, Botao Zhao, Lixia Qin, Liwei Shi, Gang Tang, Hongliang Shi, J. Appl. Phys. 114, 023514 (2013).

[27] L. Khaber, A. Beniaiche, A. Hachemi, Solid State Commun. 189, 32 (2014).

[28] R. Hill, Proc. Phys. Soc. Lond. A 65, 349 (1952).

[29] M. Born, K. Huang, Dynamical Theory of Crystal Lattices, Oxford University Press, New York 1988.

[30] A. Hachemi, H. Hachemi, A. Ferhat-Hamida, L. Louail, Phys. Scr. 82, 025602 (2010).

[31] P. Ravindran, L. Fast, P.A. Korzhavyi, B. Johansson, J. Wills, O. Eriksson, J. Appl. Phys. 84, 4891 (1998).

[32] S.F. Pugh, Philos. Mag. 45, 823 (1954).

[33] S.I. Ranganathan, M. Ostoja-Starzewski, Phys. Rev. Lett. 101, 055504 (2008).

[34] Jian Sun, Hui-Tian Wang, Nai-Ben Ming, Julong He, Yongjun Tian, Appl. Phys. Lett. 84, 4544 (2004).

[35] M. Cardona, Phys. Rev. 140A, 651 (1965). 